\title{
ANÁLISIS DE LA REFORMA EDUCATIVA EN EL ECUADOR
}

\author{
ANALYSIS OF EDUCATIONAL REFORM IN ECUADOR
}

\author{
José Alberto VÁsquez Flores * \\ Víctor Alberto Betancourt GonZaga ** \\ Gonzalo Junior Chávez Cruz *** \\ JosÉ Vicente MaZa IÑIgUeZ **** \\ Alexander Geovanny Herrera Freire ***** \\ GLADYS NARCISA ZÚÑIGA REYES ${ }^{* * * * * *}$ \\ Docentes de la Universidad Técnica de Machala - Ecuador \\ [Recepción: Setiembre de 2014/ Conformidad: Octubre 2014]
}

\section{RESUMEN}

El presente artículo está orientado a analizar la reforma educativa en el Ecuador a partir de la nueva institucionalidad del país, la que se constituye en el gobierno del Econ. Rafael Correa Delgado con el nuevo modelo educativo que se inició en enero de 2010, a partir de la reestructuración total del Ministerio de Educación, cuyo objetivo fue asegurar el camino a la educación inicial en el Ecuador con calidad y calidez, fortaleciendo la autoridad educativa, articulada con un sistema desconcentrador, que propicia un acercamiento con los ciudadanos y un acceso a la educación gratuita en todos los niveles: primaria, secundaria y superior, con algunos condicionantes necesarios.

Se redacta una introducción a partir de la reforma educativa sucedida en el año 1983, la que origina cuatro años después, la "Ley Orgánica de Educación" o Ley 127, publicada en el Registro Oficial 484 del 3 de mayo de 1983.

Este análisis contribuye también a demostrar los resultados concretos o el progreso social y educativo que ha generado la reforma educativa dentro del contexto interior y exterior, tomando como base la perspectiva del antes y después, y cómo mediante, su aplicación se ha logrado fortalecer los procesos de mejoramiento de la calidad, relevancia, equidad y eficiencia de la educación en el Ecuador.

Palabras clave: Reforma educativa; educación inicial; intercultural; institucionalidad; calidad educativa.

\begin{abstract}
The present article seeks to analyze the educational reform in Ecuador from the new institutions of the country, which constitutes the government of Econ. Rafael Correa Delgado with the new educational model that began in January 2010, after the complete restructuring of the Ministry of Education, whose objective was to ensure the path to early education in Ecuador with quality and warmth, strengthening the education authority, deconcentration articulated a system that encourages an approach with citizens and access to free education at all levels: primary, secondary and higher, with some necessary conditions. An introduction is drawn from educational reform succeeded in 1983, which originates four years later, the "Education Law" or Law 127, published in Official Record 484 of May 3, 1983.

This analysis also helps to demonstrate concrete results or social and educational progress that has generated educational reform within the internal and external environment, based on the perspective of before and after, and how through, its application has been made to strengthen the processes to improve the quality, relevance, equity and efficiency of education in Ecuador.
\end{abstract}

\section{Keywords:}

Educational reform; early childhood education; intercultural; institutions; educational quality.

\footnotetext{
* Magíster en Auditoría y Contabilidad. Email : jvasquez@utmachala.edu.ec

** Magíster en Auditoría y Contabilidad. Email : vbetancourth@utmachala.edu.ec

*** Magíster en Administración de Empresas. Email : gchavez@utmachala.edu.ec

**** Magíster en Auditoría y Contabilidad. Email : jmaza@utmachala.edu.ec

***** Magíster en Tributación y Finanzas. Email:geovamach@yahoo.es

****** Magíster en Educación Superior. Email : gladys.zr@hotmail.com
} 
José Alberto Vásquez Flores - Víctor Alberto Betancourt Gonzaga Gonzalo Junior Chávez Cruz - José Vicente Maza IÑiguez

Alexander Geovanny Herrera Freire - Gladys Narcisa Zúñiga Reyes

\section{INTRODUCCIÓN}

La última reforma educativa a nivel inicial y medio en el Ecuador fue en el año 1983, cuatro años después que volvió la democratización en el país. Con ello, la "Ley Orgánica de Educación” o Ley 127, es publicada en el Registro Oficial 484 del 3 de mayo de 1983.

Con la llegada del nuevo gobierno constitucional del Ecuador en el 2007, se propuso construir una nueva Constitución, logrando el objetivo a través de la Asamblea Constituyente, a partir del 10 de octubre de 2008.

El parámetro que ha tomado el Ecuador como base para sustentar la educación, es el Programa de Promoción de la Reforma Educativa en América Latina y el Caribe - PREAL comprometiéndose a mejorar la asignación de recursos, con el objeto de alcanzar el 6\% del Producto Interno Bruto - PIB ${ }^{1}$.

Tuvieron que pasar 28 años desde la promulgación de la última ley educativa, para expedir la nueva Ley Orgánica de Educación Intercultural, que se publicó en el Registro Oficial 417 de 31 de marzo de 2011.

La Constitución de la República en su Art. 26, reconoce a la educación como un derecho que las personas ejercen a lo largo de su vida y un deber ineludible e inexcusable del Estado, garantía de la igualdad e inclusión social.

La Constitución de la República en su Art. 27, establece que la educación debe estar centrada en el ser humano y garantizar su desarrollo holístico, en el marco del respeto a los derechos humanos, al medio ambiente sustentable y a la democracia participativa, obligatoria, intercultural, incluyente y diversa, de calidad y calidez; impulsando la equidad de género, la justicia, la solidaridad y la paz; estimulando también el sentido crítico, el arte y la cultura física, la iniciativa individual y comunitaria, y el desarrollo de competencias y capacidades para crear y trabajar.

La Constitución de la República en su Art. 28, establece que la educación responderá al interés público y no estará al servicio de intereses individuales y corporativos. Se garantizará el acceso universal, permanencia, movilidad y egreso sin discriminación alguna y la obligatoriedad en el nivel inicial, básico y bachillerato o su equivalente.

La Constitución de la República en su Art. 29, declara que el Estado garantizará la libertad de enseñanza, y el derecho de las personas de aprender en su propia lengua y ámbito cultural.

La Constitución de la República, en su Art. 286 establece que las finanzas públicas, en todos los niveles de gobierno, se conducirán de forma sostenible, responsable y transparente, y procurarán la estabilidad económica. Los egresos permanentes se financiarán con ingresos permanentes. Los egresos permanentes para educación serán prioritarios y, de manera excepcional, podrán ser financiados con ingresos no permanentes.

La Constitución de la República, en su Art. 343 establece un sistema nacional de educación que tendrá como finalidad el desarrollo de capacidades y potencialidades individuales y colectivas de la población, que posibiliten el aprendizaje, y la generación y utilización de conocimientos, técnicas, saberes, artes y cultura.

La Constitución de la República, en su Art. 344 dicta que el sistema nacional de educación comprenderá las instituciones, programas, políticas, recursos y actores del proceso educativo, así como acciones en los niveles de educación inicial, básica y bachillerato, y estará articulado con el sistema de educación superior.

La Constitución de la República, en su Art. 349 establece que el Estado garantizará al personal docente, en todos los niveles y modalidades, estabilidad, actualización, formación continua y mejoramiento pedagógico y académico; una remuneración justa, de acuerdo a la profesionalización, desempeño y méritos académicos.

Con estas normativas de la Constitución de la República del Ecuador, se expide la "LEY ORGÁNICA DE EDUCACIÓN INTERCULTURAL”, la cual toma como base sustentable, la educación como 
herramienta indispensable para el conocimiento, constituyéndose en un eje estratégico para el desarrollo nacional.

Además, en la Constitución de la República del Ecuador, en su disposición transitoria décimo octava establece que el Estado asignará de forma progresiva recursos públicos del Presupuesto General del Estado para la educación inicial, básica y bachillerato, con incrementos anuales de al menos el cero punto cinco por ciento $(0,5 \%)$ del Producto Interno Bruto hasta alcanzar un mínimo de seis por ciento (6\%) del Producto Interno Bruto.

\section{UN NUEVO MARCO PARA EL ANÁLISIS DE LA REFORMA EDUCATIVA}

El nuevo modelo educativo se inició en enero de 2010, con la reestructuración total del Ministerio de Educación, con el objetivo de asegurar el acceso a la educación inicial en el Ecuador con calidad y calidez fortaleciendo la autoridad educativa, articulado con un sistema desconcentrador. Para cumplir con este objetivo se ha apostó por la desconcentración cuyo fin es buscar un acercamiento con los ciudadanos. Hoy el Ministerio cuenta con un nuevo órgano funcional y agrupa a las provincias del Ecuador en 9 zonas claramente definidas.

\begin{tabular}{|c|c|}
\hline ZONAS & PROVINCIAS \\
\hline Zona 1 & Esmeraldas, Carchi, Imbabura, Sucumbíos \\
\hline Zona 2 & Pichincha, Napo y Orellana \\
\hline Zona 3 & Cotopaxi, Tungurahua, Chimborazo, \\
Pastaza
\end{tabular}

Fuente: Ministerio de Educación
Bajo este esquema se conforman Direcciones Regionales y Distritales que crean circuitos de educación y gobiernos escolares, dotándoles de sus propios sistemas de información.

Por otra parte, la Ley Orgánica de Educación Intercultural (LOEI) en su Art. 25 señala que los niveles de autoridad educativa nacional están formados por cuatro niveles (Ministerio, Direcciones Regionales, Direcciones Distritales y Gobiernos Escolares), que garantizan plenamente los derechos a la educación; su nivel central se encarga de la rectoría, regulación y control; el nivel zonal intercultural y bilingüe es el ente encargado de coordinar las actividades y asegurar la calidad y trámites con atención a ciudadanos, además, se le encarga la ejecución de los servicios educativos a los ciudadanos de todas las nacionalidades y pueblos del país fomentando la unidad en la diversidad donde se desarrollen las habilidades básicas y específicas para potenciar el desarrollo cultural y socioeconómico del país.

Bajo esta óptica se pretende que para el año 2031 se conozcan los resultados de estos positivos cambios y podamos contar con profesionales de nuestro país, con nuevas competencias y sobre todo con formación en valores, que serán quienes generen los anhelados cambios en el Ecuador.

\section{PROPÓSITOS DE LA REFORMA EDUCATIVA}

La principal motivación de la reforma educativa es propiciar y potencializar los factores de calidad, equidad, interculturalidad y universalidad. De este modo, se detallan los siguientes propósitos específicos:

- Propiciar la sensibilización de la sociedad nacional y de las comunidades locales con la educación. Si la educación no es vista y sentida como una cuestión que atañe a todos, difícilmente creará inclusión en los procesos y en las necesarias transformaciones del país ${ }^{2}$.

- Mejorar la calidad educativa, que implica terminar con la selectividad, hacer pruebas finales nacionales al fin de cada etapa y reforzar la formación en lengua y matemáticas, entre otros cambios ${ }^{3}$. 
- También se pretende mejorar la Educación para la ciudadanía y permitir a las administraciones que puedan concertar la financiación pública de colegios de enseñanza sin considerar género ${ }^{4}$.

- Detener el abandono y el fracaso escolar.

- Preparar a los estudiantes para la vida, para la participación en una sociedad democrática, para el mundo laboral o de emprendimiento y para continuar con sus estudios universitarios ${ }^{5}$.

Todos estos propósitos tienen íntima relación con los objetivos que se propone el gobierno central a través del Ministerio de Educación.

\section{ALGUNOS RESULTADOS CONCRETOS DENTRO DEL CONTEXTO}

La educación se ha convertido en la actualidad en la piedra angular en torno al desarrollo y al crecimiento económico y social de las naciones; por esta razón, los países desarrollados han realizado cuantiosas inversiones para incrementar la cobertura y calidad de su educación. En el mundo industrializado, la adecuación de estándares educativos al contexto de país o estamento institucional se considera estratégico, lo que es motivo de análisis y discusión permanente.

Además, es frecuente analizar los días al año y el número de horas que dedica cada alumno a estudiar en cada Región.

Es así que mientras el año escolar tiene 243 días en Japón, 220 en Corea del Sur, 216 en Israel, 200 en Holanda y Tailandia, en los países Latinoamericanos, es de tan solo 160 días y en los sectores rurales alcanza apenas 130 días. En el Ecuador a partir del año 2011 se incrementó a 210 días.

Los niños Chinos y los de Singapur estudian de 12 a 14 horas al día en el horario regular y luego, asisten a centros de tutoría privados; los niños de Latinoamérica no llegan a estos parámetros y particularmente, los ecuatorianos solo dedican a la educación 6 horas diarias.

Lamentablemente en nuestro país, el mejoramiento de la calidad educativa no ha tenido la atención necesaria, debido a que los diferentes gobiernos

4 http://unesdoc.unesco.org/images/0014/001446/144666s.pdf 5 http://educacion.gob.ec/bachillerato-general-unificado/ democráticos que han pasado, siempre han preferido invertir en la obra pública visible, que pueda estar a la vista de todos frente a las elecciones más próximas, a invertir en el mejoramiento educativo o en la obra que no es visible, que no produce resultados electorales, pero sí resultados a futuro o alrededor de cinco, diez o veinte años.

El gobierno de la revolución ciudadana liderado por el Econ. Rafael Correa Delgado, ha emprendido en el Ecuador una reforma educativa que busca una economía del talento y dejar de ser una economía de recursos limitados como hasta ahora; es decir, dejar de depender de productos agrícolas y energéticos, para convertirse en una economía de las ideas y el talento humano, tomando en cuenta que el gobierno considera a la educación como un "bien público que pertenece a todos los ecuatorianos, por el impacto que esto tiene dentro de la sociedad".

Evidentemente la clave para la reducción de la pobreza y el desarrollo sostenible de un país, no es solamente la economía, sino una educación de calidad, partiendo desde la educación inicial.

Mientras todos seguimos pendientes de lo que desarrolla la política gubernamental, el futuro de nuestros países debe ser visto desde la perspectiva de una educación de calidad, desde su inicio.

Este es un análisis que se realiza tomando en consideración un estudio hecho por el Programa de Promoción de la Reforma Educativa en América Latina y el Caribe (PREAL), fundación Ecuador y el grupo de investigación y acción para el bienestar público presentado en el año 2010.

El estudio realizado por estos organismos ha sido de gran interés por cuanto se hacen comparaciones con otros estudios realizados en años anteriores y la forma como ha evolucionado el sistema educativo hasta el año 2009, y se analiza las perspectivas que tienen hasta llegar al 2020, con la implementación del Plan Decenal de Educación.

De acuerdo a este plan, podemos decir que el sistema educativo ha tenido un avance desde que se dio el penúltimo informe en el año 2006; pues los países de América Latina se han comprometido a mejorar 
en cuanto a la asignación de recursos, teniendo como objetivo otorgar el 6\% del PIB (Producto Interno Bruto) para la educación.

Del 6\% para educación del PIB descrito por el PREAL, en el Ecuador se ha superado esta meta; es así que, en el año 2012 se alcanzó el 6,8\% y en el año 2013 se mantuvo en el 6,5\%. El gobierno central deberá mantener este nivel de inversión en la educación, evaluando resultados, comparando cuántos bachilleres han accedido a la educación superior y cuántos han llegado a culminar el tercer nivel, lo que nos indicará si la reforma ha tenido un impacto positivo.

Se planteó un nuevo sistema de evaluación, en el que no solo se evalúa a los estudiantes; sino también, a profesores y a la gestión gubernamental, lo cual ha permitido tener un criterio más amplio, real y objetivo de la realidad educativa en América Latina $y$, particularmente, en el Ecuador.

Sin embargo, una vez cumplida la recomendación hecha por el PREAL, la educación en el Ecuador todavía necesita grandes cambios que se deben plantear como desafíos: terminar con el analfabetismo cultural y social; atender a un proyecto destinado a alcanzar que la persona pueda llegar a realizar razonamientos lógicos, encaminándose por la senda de la reflexión lógica y analítica.

\section{PROGRAMAS QUE SE IMPLEMENTARON COMO APOYO AL ESTUDIANTE}

Eliminación del Aporte Voluntario.- Anteriormente, los padres de familia se veían obligados a realizar un aporte de 25,00 USD para cubrir costos adicionales de profesores, comprar material y realizar mantenimiento. Desde el año 2007, el Ministerio de Educación asumió este valor como uno de sus programas de eliminación de barreras de acceso a la escolaridad.
Programa de Alimentación Escolar.- Este programa otorgaba desayunos y almuerzos a estudiantes de educación general básica de escuelas públicas, en zonas urbanas marginales y rurales con mayor vulnerabilidad social. Como por ejemplo, en el año 2008, fueron 1385964 los estudiantes beneficiados ${ }^{6}$

Hilando el Desarrollo.- En el año 2008, el Ministerio de Educación, junto con el Ministerio de Coordinación de Desarrollo Social, entregaron 715427 uniformes escolares gratuitos a los estudiantes de la zona rural ${ }^{7}$.

Textos Escolares.- Después de un proceso de selección y corrección de los textos escolares en el año 2007, el Ministerio de Educación empezó la entrega de textos escolares gratuitos en el año 2008. En ese mismo año, 2996399 estudiantes de "educación general básica" recibieron textos escolares gratuitos para su educación ${ }^{8}$.

Tabla No 01:

Programas Sociales Específicos en Educación Año 2009

\begin{tabular}{|c|c|}
\hline Proyecto & $\begin{array}{c}\text { Presupuesto } \\
\text { ejecutado en USD }\end{array}$ \\
\hline Hilando al desarrollo & 13700000,00 \\
\hline Textos escolares gratuitos & 11600000,00 \\
\hline $\begin{array}{c}\text { Eliminación de aporte } \\
\text { voluntario }\end{array}$ & 12400000,00 \\
\hline $\begin{array}{c}\text { Alfabetización y educación } \\
\text { compensatoria }\end{array}$ & 4800000,00 \\
\hline Educación Inicial & 900000,00 \\
\hline $\begin{array}{c}\text { Programa de alimentación } \\
\text { escolar }\end{array}$ & 32200000,00 \\
\hline Total programa sectorial & 75600000,00 \\
\hline Total sectorial educación & 2071300000,00 \\
\hline Participación de los programas & $3,65 \%$ \\
\hline
\end{tabular}

Fuente: http://www.unicef.org/ecuador/como_va_la_inversion_n28_Parte1.pdf

Elaboración propia.

6 http://www.elmercurio.com.ec/280405-reforma-educativa-impostergable/\#.U4QdBdJ5Mg0

7 Ibíd.

8 Ibíd 
Gráfico No 01:

Presupuesto ejecutado

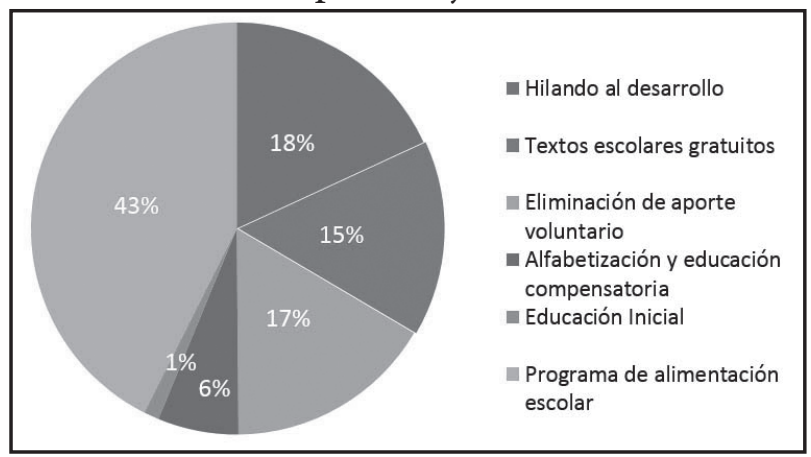

Elaboración propia.

Como se puede observar en la tabla $\mathrm{N}^{\circ} 01 \mathrm{y}$ gráfico $\mathrm{N}^{\circ} 01$, el porcentaje más alto de los programas sociales específicos de la educación, es el de la alimentación escolar que representa un $43 \%$; en segundo lugar, le sigue hilando al desarrollo, con un $18 \%$; en tercero la absorción por la eliminación del aporte voluntario, con el 17\%; en cuarto los textos escolares gratuitos, con el 15\%; en quinto la alfabetización y educación compensatoria, con el 6\%; y del $1 \%$ la educación inicial.

De la tabla 1 , se desprende una aplicación del $3,65 \%$ de la participación de los programas sobre el total sectorial de la educación.

\section{Gráfico No 02:}

Porcentaje de estudiantes de 5 a 14 años, que asisten al primero de básico y al sistema escolar

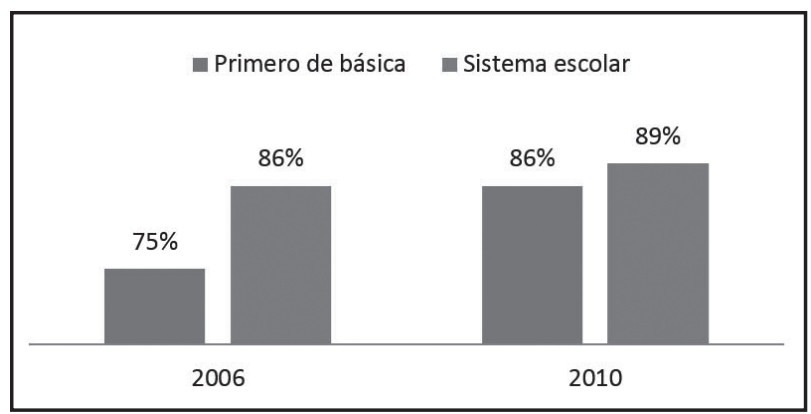

Fuente: http://www.unicef.org/ecuador/como_va_la_inversion_n28_Parte1.pdf

Elaboración propia.

Como se puede apreciar en el gráfico $\mathrm{N}^{\circ} 2$, una vez eliminada esta erogación económica, el acceso a la educación totalmente gratuita, se ha visto reflejado en el incremento de estudiantes de 5 a 14 años en el año 2006 de primero de básica del $75 \%$ al $86 \%$ en el
2010; y, en el sistema escolar en el 2006 del $86 \%$ al $89 \%$ en el 2010.

\section{Gráfico No 03:}

Porcentaje de estudiantes de 15 a 17 años, que asisten al primero de básica y al sistema escolar

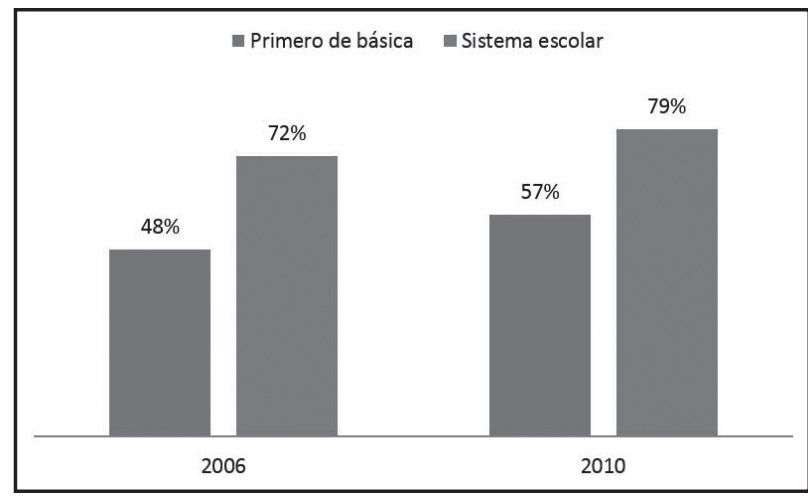

Fuente: http://www.unicef.org/ecuador/como_va_la_inversion_n28_Parte1.pdf Elaboración propia.

Como se puede apreciar en el gráfico $\mathrm{N}^{\circ} 03$, una vez eliminada esta erogación económica, el acceso a la educación totalmente gratuita, se ha visto reflejado con el incremento de estudiantes de 15 a 17 años. En el año 2006 de primero de básica del $48 \%$ al 57\% en el 2010; y, en el sistema escolar en el 2006 del $72 \%$ al 79\% en el 2010.

Del análisis de los gráficos presentados, se desprende que estos programas que se implementaron como apoyo al estudiante, ha tenido un efecto positivo. La reforma educativa en el Ecuador está en marcha, solo falta el compromiso de seguir trabajando juntos en beneficio de este proyecto.

Los cambios trascendentales que ha emprendido el Gobierno del Ecuador referente a la reforma educativa, permite avizorar la llegada al desarrollo y al buen vivir. En este contexto, la educación con los programas en el nivel inicial, básico y bachillerato, deberán ser medibles durante del tiempo que se van desarrollando las competencias en sus educandos.

En general, los resultados de las reformas educativas se obtienen en generaciones posteriores. Sin embargo, en el Ecuador estas reformas están evidenciando avances en cada etapa, lo cual nos permite avizorar el cumplimiento de los objetivos y metas propuestas. 


\section{CONCLUSIONES}

1. La reforma de la educación ecuatoriana, ha sido creada buscando contribuir con una función social de trascendencia a la actual sociedad denominada del conocimiento.

2. El propósito de la reforma educativa está orientado a la educación de calidad, pertinencia y calidez sustentada en valores, que conlleve a la formación de la persona, no sólo para el trabajo y la competencia, sino y sobre todo, para el buen vivir en comunidad.

3. La reforma educativa abarca desde el nivel inicial, básico y bachillerato, integrándola como parte estratégica del desarrollo sostenible del país.

4. La inversión en educación ha alcanzado en el 2011 el 6\% del PIB, para el 2012, alcanzó el 6.8\% y en el 2013 se mantuvo en el 6,5\%. Ecuador ha cumplido con el parámetro prestablecido por el PREAL, sobrepasando el 6\% de educación del PIB, sosteniendo lo propuesto por el gobierno ecuatoriano.

\section{REFERENCIAS BIBLIOGRÁFICAS}

1. Registro Oficial 449, Constitución de la República del Ecuador, año 2008.

2. Registro Oficial 417, Ley Orgánica de Educación Intercultural, año 2011.

\section{REFERENCIAS ELECTRÓNICAS}

1. http://jpincay 1974 .wordpress . com/2011/05/23/primer-analisis-de-la-educacion-en-el-ecuador/

2. http://www.elmercurio.com.ec/280405-reforma-educativa-impostergable/\#.U4QdBdJ5Mg0

3. http://educacion.gob.ec/desconcentracion-del-mineduc/.Ley Orgánica de Educación Intercultural

4. http://www.cepal.org/publicaciones/xml/

5. http://www.uasb.edu.ec/UserFiles/372/File/ pdfs/REFORMA\%20DEL\%20BACHILLERATO/DECRETO-EJECUTIVO-1786.pdf

6. http://www.boe.es/boe/dias/2014/03/01/ pdfs/BOE-A-2014-2222.pdf

7. http://educacion.gob.ec/bachillerato-general-unificado/ 